\title{
An Improved Formula for Lamination Core Loss Calculations in Machines Operating with High Frequency and High Flux Density Excitation
}

\author{
Yicheng Chen \\ Department of Electrical \& Computer Engineering \\ Clarkson University, Potsdam, NY 13699-5720, USA \\ Also, Shenyang University of Technology, P.R.China \\ yicheng@clarkson.edu
}

\author{
Pragasen Pillay, Senior Member, IEEE \\ Department of Electrical \& Computer Engineering \\ Clarkson University \\ Potsdam, NY 13699-5720, USA \\ pillayp@clarkson.edu
}

\begin{abstract}
For electrical machine designers, core loss data are usually provided in the form of tables or curves of total loss versus flux density or frequency. These can be used to extract the loss coefficients of the core loss formulas. In this paper, three currently available formulas are discussed and compared with the loss data supplied by lamination steel manufacturers. It is found that the dynamic hysteresis loop plays an important role in the total loss calculation, especially at high flux densities and high frequencies, and the loss coefficients should change with frequency. A new modified formula is proposed to represent the coefficient changes. The new curve is applied to the measured manufacturer's data, with acceptable accuracy.
\end{abstract}

Keywords-electrical machines; hesteresis loss; eddy current loss; core loss; excess loss; high frequency excitation; high flux density excitation.

\section{INTRODUCTION}

Core loss in a magnetic material occurs when the material is subjected to a time varying magnetic flux. The actual physical nature of this loss is still not completely understood and a simplistic explanation of this complex mechanism is as follows. Energy is used to effect "magnetic domain wall motion" as the domains grow and rotate under the influence of an externally applied magnetic field. When the external field is reduced or reversed from a given value, domain wall motion again occurs to realize the necessary alignment of domains with the new value of the magnetic field. The energy associated with domain wall motion is irreversible and manifests itself as heat within the magnetic material. The rate at which the external field is changed has a strong influence upon the magnitude of the loss, and the loss is generally proportional to some function of the frequency of variation of the magnetic field. The metallurgical structure of the magnetic material, including its electrical conductivity, also has a profound effect upon the magnitude of the loss. In electrical machines, this loss is generally termed the core loss.

Traditionally, core loss $P_{c}$ has been divided up into two components: hysteresis loss $P_{h}$ and eddy current loss $P_{e}$.
According to the Steinmetz equation, measurement and calculation of core losses are normally made with sinusoidal flux density of varying magnitude and frequency. These measurements and calculations are based on the standard coil and frequently modeled by a two term function of the form

$$
\begin{aligned}
P_{c} & =P_{h}+P_{e} \\
& =k_{h} f B^{n}+k_{e} f^{2} B^{2},
\end{aligned}
$$

where $f$ is the frequency of the external magnetic field, $B$ is flux density, $k_{h}, k_{e}$ and $n$ are the coefficients, which depend on the lamination material, thickness, conductivity, as well as other factors. However, this formula is only applicable under the assumption that the maximum magnetic flux density of 1.0 Tesla is not exceeded and the hysteresis loop is under the static situation, which is not practical in electrical machines. When the magnetic flux density is over 1.0 Tesla or the field frequency becomes high, there is a big discrepancy between the calculation based on (1) and experimental results.

For correction and modification, various models have been proposed [5], [6], [9], [10], [12], using the domain wall theory to explain the core loss. While such models provide useful insights into the loss mechanism, they do not, as yet accurately account for the so-called "anomalous loss", or excess loss in a real lamination specimen, nor do they consider the flux harmonics. One of the modifications [21] for (1) is

$$
\begin{aligned}
P_{c} & =P_{h}+P_{e} \\
& =k_{h} f B^{(a+b B)}+k_{e} f^{2} B^{2},
\end{aligned}
$$

where $a$ and $b$ are constant, and the exponent coefficient of hysteresis loss is supposed to change linearly with flux density.

As the core loss plays an increasingly important role both in the improvement of the quality of electrical steels at the production stage and in the optimization of their operating 
conditions, the research of the physical understanding of the dependence of core losses on peak magnetic induction, magnetizing frequency and microstructure in soft magnetic materials is ongoing [1]-[4]. Progress in this direction has been achieved in recent years by a statistical loss theory [16], which provides a physical justification for separation of losses into hysteresis, eddy, and excess contributions, and shows that the origin of the excess loss can be well understood by describing the magnetization dynamics in terms of a random distribution of magnetic correlation regions (i.e., groups of interacting domain walls), termed magnetic objects (MO). It assumes that the excess loss is governed by the statistical distribution of the local threshold fields at which different MOs become magnetically active. According to this theory, the core loss under sinusoidal flux condition is given by

$$
\begin{aligned}
P_{c} & =P_{h}+P_{e}+P_{a} \\
& =k_{h} f B^{n}+k_{e} f^{2} B^{2}+k_{a} f^{1.5} B^{1.5},
\end{aligned}
$$

where $P_{a}$ is referred to as the excess loss and $k_{a}$ is related to the material thickness, cross-sectional area, conductivity and a parameter which describes the material microstructure. Formula (3) is in good agreement with several loss experiments, but it does not seem to be sufficiently general [8]. Deviations have been observed, resulting in the $k_{a}$ being not truly a constant, as would be expected, but exhibits a dependence on flux density.

The other factors which influence the calculation of core losses, such as the difference between the alternating core loss and the rotating core loss [1], flux distortion [19], flux waveform shape, as well as the stress in the stack are still under investigation. The continued efforts on further understanding of the physical nature of the core loss and accurate prediction of core loss in electrical machine designs are ongoing.

In practice, the lamination steel manufacturers do not present the loss coefficients mentioned above. They only provide the loss curves and tables in watts per kilogram $(\mathrm{W} / \mathrm{kg}$ ) or watts per pound (W/lb) versus flux density or frequency to indicate the combination of hysteresis loss, eddy current loss and excess loss for the design of electrical machines. The classical estimation of core loss is systematically lower than the measured values. Ratios of two or more are often observed and used as empirical and corrective parameters for machine designs. This underestimation of core loss is due to the waveform distortion, to the complexity of the electrical machine structures and to the complex behavior of dynamic hysteresis loops. A good knowledge of core loss is required to improve the following two points in the estimation process: firstly, one needs to be able to evaluate magnetic field evolution at any point of the complex electrical machine structure, and secondly, one needs to develop a dynamic loss model for changing flux densities and frequencies. Thus, for the analysis and calculation of core loss, the definition of the loss coefficients based on the original loss data or curves provided by the lamination steel manufacturers and actual experiment of a specific electrical machine becomes essential.

In this paper, loss separation for the three known loss formulas is discussed, and the loss coefficients are extracted based on the experimental data and the original loss data supplied by the steel manufacturers. Though these coefficients are obtained mathematically by polynomial curve fitting, they can be explained physically. The discrepancies between the calculation results of the three formulas and the original loss data emphasize the fact that the static hysteresis loop and the dynamic hysteresis loop are different, and the loss coefficients should change with frequency. The experimental loss data of several electrical steels, including cold rolled motor lamination steels, non-oriented silicon steels, thin-gauge silicon steels, powdered metal alloys and both annealed and un-annealed materials from the steel suppliers are used to support the discussion. A modified core loss formula with three terms indicating the hysteresis loss, eddy current loss and excess loss is developed. This formula reveals the difference between the static hysteresis loop and the dynamic hysteresis loop, which results in the dependence of the loss coefficients on frequencies. The agreement between calculations using this formula and the experimental loss data is acceptable.

\section{SEPARATION OF CORE LOSSES}

The core loss data provided by steel manufacturers or from experiments are usually in the form of curves or tables of total loss vs. flux density at different frequencies, or total loss vs. frequency at different flux densities. When divided by frequency $f$, (1) becomes

$$
\begin{aligned}
P_{c} / f & =P_{h} / f+P_{e} / f \\
& =k_{h} B^{n}+k_{e} f B^{2},
\end{aligned}
$$

which is a linear equation in frequency at each flux density $B$. Now, the core loss data are used to plot curves of $P_{c} / f$ vs. $f$ for different values of flux density $B$ from the lowest frequency to the highest frequency. The curves should be straight lines and can be represented by

$$
P_{c} / f=D+E f \text {. }
$$

The intercept $D$ on the vertical axis $\left(P_{c} / f\right)$ must be the hysteresis energy loss and equal to

$$
D=k_{h} B^{n}
$$

The intercepts $D_{1}$ and $D_{2}$ for the selected two values of flux density $B_{1}$ and $B_{2}$ are substituted into the logarithm of (6), giving two simultaneous linear algebraic equations for $k_{h}$ and $n$ of the form 


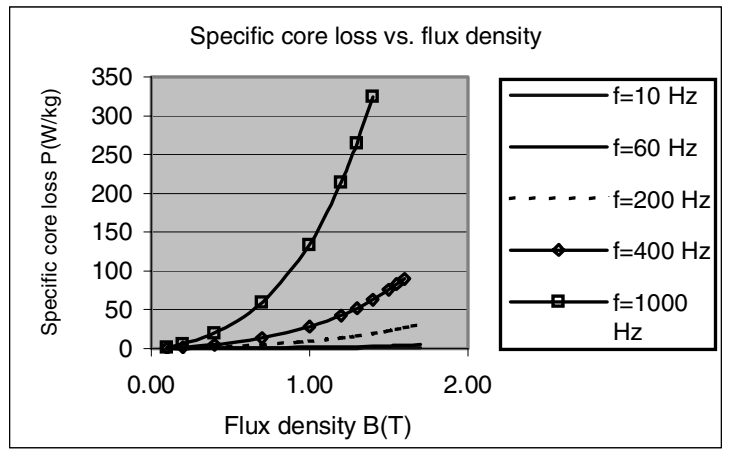

Fig. 1 Specific core loss versus flux density for Ak 26-M47

$\log D_{1}=\log k_{h}+n \log B_{1}$,

$\log D_{2}=\log k_{h}+n \log B_{2}$.

These are solved for $\log k_{h}$ and $n$, from which $k_{h}$ is obtained from $\log k_{h}$. Next, two values of $k_{e}$ are obtained from the gradients $E_{1}$ and $E_{2}$ of the two curves of $P_{c} / f$ vs. $f$. The average or the highest value can be taken as $k_{e}$.

In the same way, the loss coefficients in (2) can be obtained.

When divided by frequency $f$, (3) will become

$$
\begin{aligned}
P_{c} / f & =P_{h} / f+P_{e} / f+P_{a} / f \\
& =k_{h} B^{n}+k_{a} \sqrt{f} B^{1.5}+k_{e} f B^{2} .
\end{aligned}
$$

Here, the core loss data is used to plot curves of $P_{c} / f$ vs. square root of frequency $\sqrt{f}$, not frequency $f$, for different values of flux density $B$ from the lowest frequency to the highest frequency. These curves should be parabolas and can be represented by

$$
P_{c} / f=D+G \sqrt{f}+E(\sqrt{f})^{2},
$$

where the coefficients $D, E$ and $G$ can be obtained by polynomial curve fitting, with $P_{c} / f$ on the vertical axis and $\sqrt{f}$ on the horizontal axis. By comparing (9) and (10), we have $D=k_{h} B^{n}, G=k_{a} B^{1.5}$ and $E=k_{e} B^{2}$. Therefore, for given flux densities, the loss coefficients $k_{h}$, $n, k_{a}$ and $k_{e}$ can be obtained.

\section{MEASUREMENT AND CALCULATION RESULTS}

Several sets of measured steel loss data are used to investigate the validity of the three loss formulas (1), (2) and

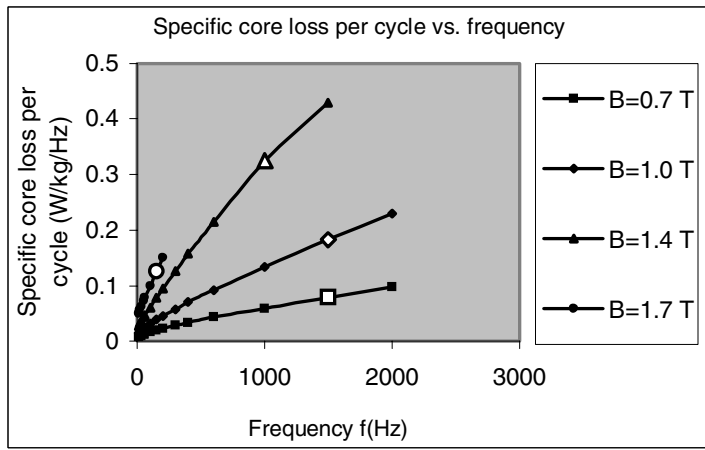

Fig. 2 Specific core loss per cycle versus frequency for Ak 26-M47

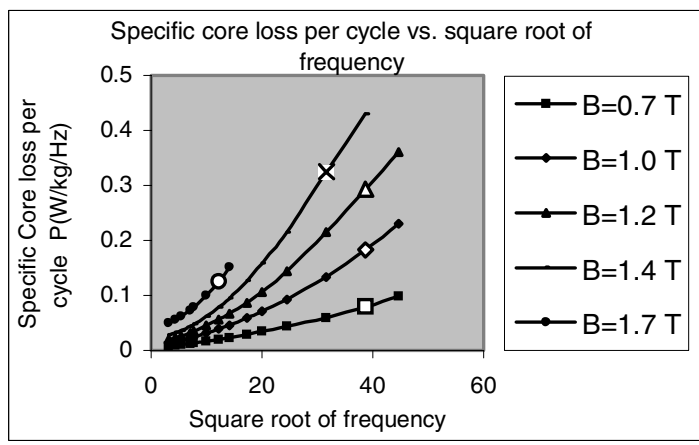

Fig. 3 Specific core loss per cycle versus square root of frequency for Ak 26-M47

(3), including cold rolled motor lamination steels, nonoriented silicon steels, thin-gauge silicon steels, powdered metal alloys and both annealed and un-annealed materials. As an example, Fig. 1 shows the core loss versus magnetic flux density for AK 26-M47 provided by the steel material manufacturer. Fig. 2 shows the curves of core energy loss $P_{c} / f$ versus frequency $f$ of AK $26 \mathrm{M}-47$, and Fig. 3 shows the curves of core energy loss $P_{c} / f$ versus $\sqrt{f}$ of AK 26M47.

Fig. 4 through to Fig. 9 show the comparisons between the original loss data and the calculations based on (1), (2) and (3) for AK 26-M47 respectively.

\section{DISCUSSION OF RESULTS}

It can be seen that there is a deviation between the original loss data and the calculation based on formula (1). The agreement between the original loss data and the calculation in (2) at $60 \mathrm{~Hz}$ is much better than that in (1). The best situation at $60 \mathrm{~Hz}$ is shown in Fig. 8 for (3). All the calculation results based on the three formulas (1), (2) and (3) at $400 \mathrm{~Hz}$ have large deviations from the original loss data. The errors at saturation flux densities are over thirty percent. The coefficients used for the comparisons are the same for different frequencies in the same formula, but different between the formulas. 


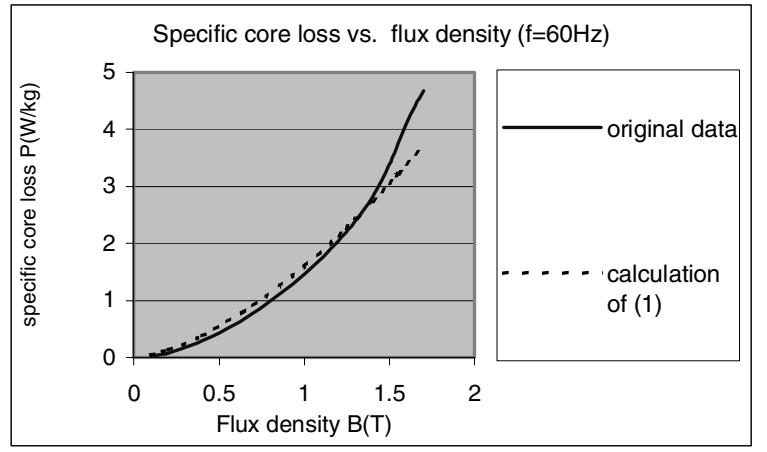

Fig.4 Comparison between the original loss data and the calculation of formula (1) for Ak 26-M47 $(60 \mathrm{~Hz})$

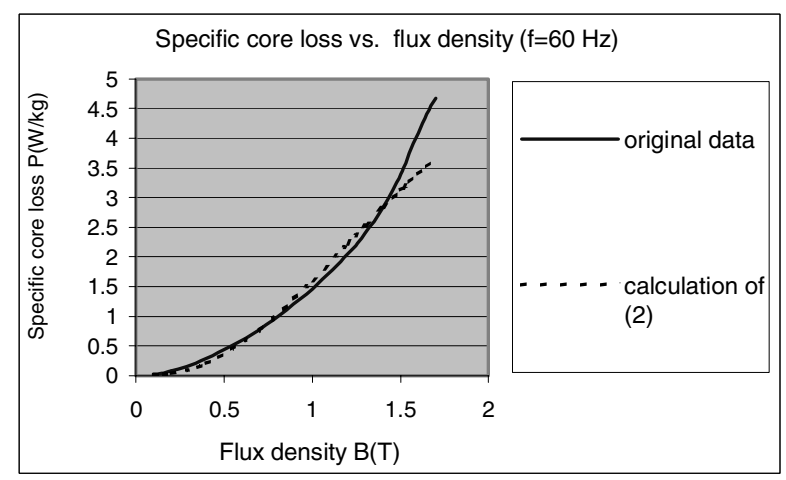

Fig.5 Comparison between the original loss data and the calculation of formula (2) for Ak 26-M47 $(60 \mathrm{~Hz})$

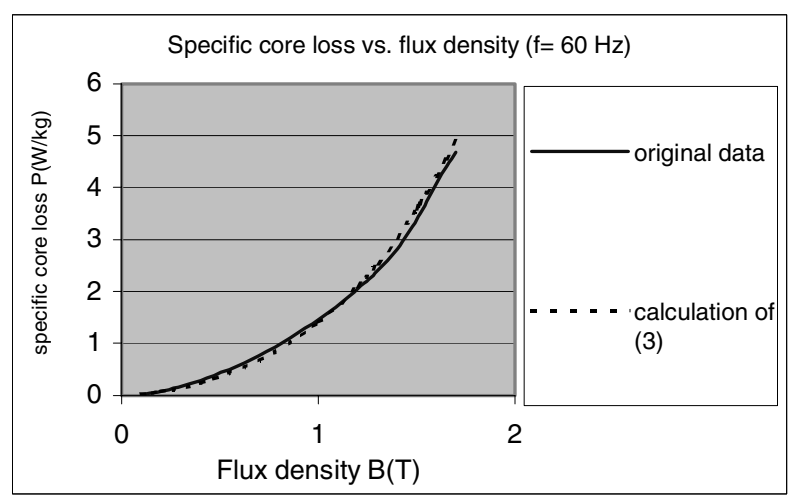

Fig.6 Comparison between the original loss data and the calculation of formula (3) for Ak 26-M47 $(60 \mathrm{~Hz})$

At the lower flux densities, the calculations from the three formulas have good agreement with the actual loss data. But at the higher flux densities over 1.2 Tesla, larger discrepancies are observed. On one hand, this is because the models, on which the three formulas are developed, have assumptions of low flux densities. On the other hand, this indicates that the coefficients are not constant when frequency and changes need to be incorporated at high flux densities.

As can be seen in Fig. 2, at higher flux densities, the curves of core loss versus frequency are not straight lines as predicted. Similarly, the curves of core loss versus square root of frequency in Fig. 3 are not parabolas at high flux densities.

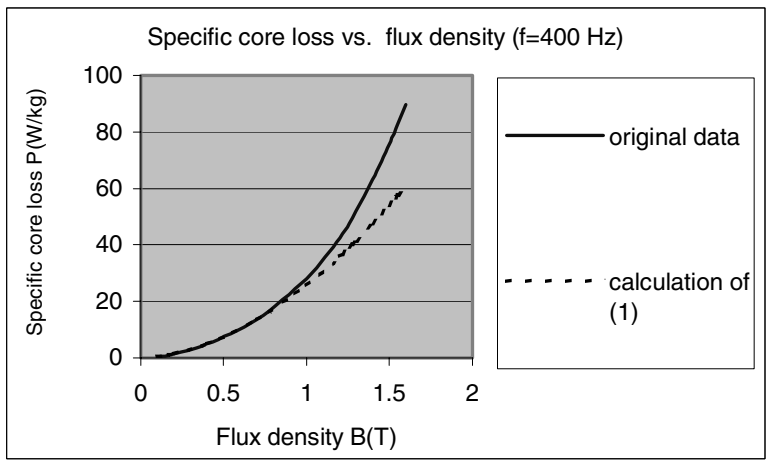

Fig.7 Comparison between the original loss data and the calculation of formula (1) for Ak 26-M47 $(400 \mathrm{~Hz})$

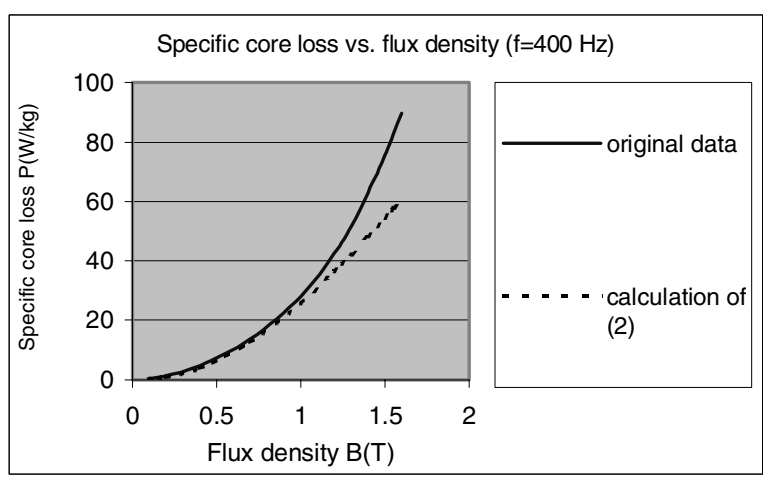

Fig.8 Comparison between the original loss data and the calculation of formula (2) for Ak 26-M47 $(400 \mathrm{~Hz})$

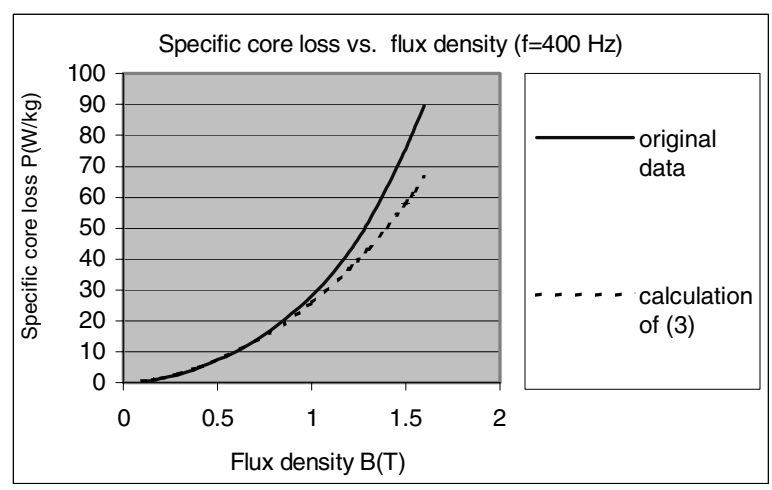

Fig.9 Comparison between the original loss data and the calculation of formula (3) for Ak 26-M47 $(400 \mathrm{~Hz})$

Several reports have shown the same anomalous non-linear behavior of the core loss versus frequency [13], [15], [18], [20], but no reports on the parabola of core loss versus the square root of frequency are found. These results show that the models for the three formulas are not complete for the whole range of flux density and frequency.

Formula (1) is less accurate because of its static Preisach model and does not include the excess loss. Formula (2) is a modification of formula (1). Mathematically, (2) has better curve fitting because more data is used to extract the coefficients in (2) than in (1). 


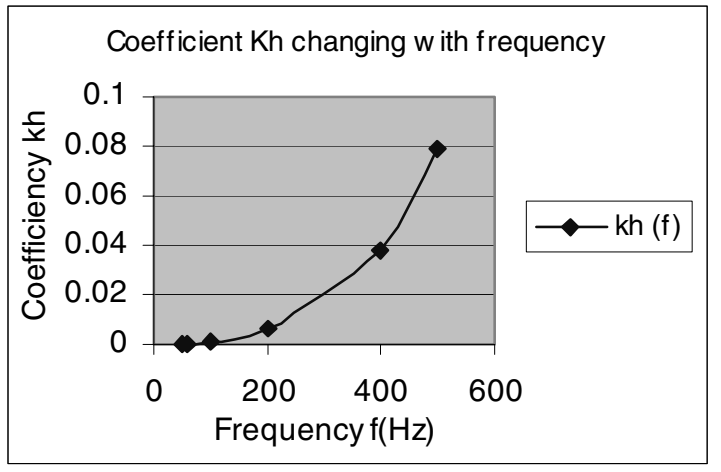

Fig.10 Coefficient $k_{h}$ changing with frequencies

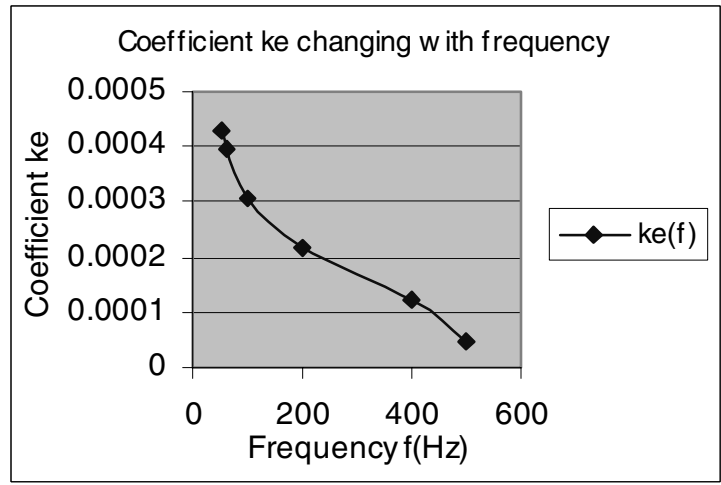

Fig. 11 Coefficient $n$ changing with frequencies

The three-term model, which has resulted in (3), indicates the presence of the excess loss, using domain wall and statistical theory. This model has been generally accepted and confirmed by different researches. The static hysteresis loop is used to calculate the hysteresis loss, and the skin effect is often ignored. Indeed, under low flux densities and low frequencies, this model has achieved good agreement with experimental data. However, significant discrepancies between the calculation results of this model and the experimental data occur under high flux densities and high frequencies. Actually, the static hysteresis loop and the dynamic hysteresis loop are different. And when the flux density is in saturation; the hysteresis loop will change shape. At high frequencies, the skin effect also becomes important. Therefore, it is natural that there is a discrepancy between the calculation results of (3) and the experimental data at high flux densities and high frequencies, as it uses a static hysteresis loop instead of the dynamic hysteresis loop to calculate the hysteresis loss and ignores the skin effect in the eddy current loss calculation. This discrepancy also indicates that the coefficients are not constant when the frequency changes.

To further investigate the coefficient changes with frequency, the coefficients $k_{h}, k_{e}$ and $n$ are plotted, while the excess loss coefficient $k_{a}$ is supposed to be unchangeable

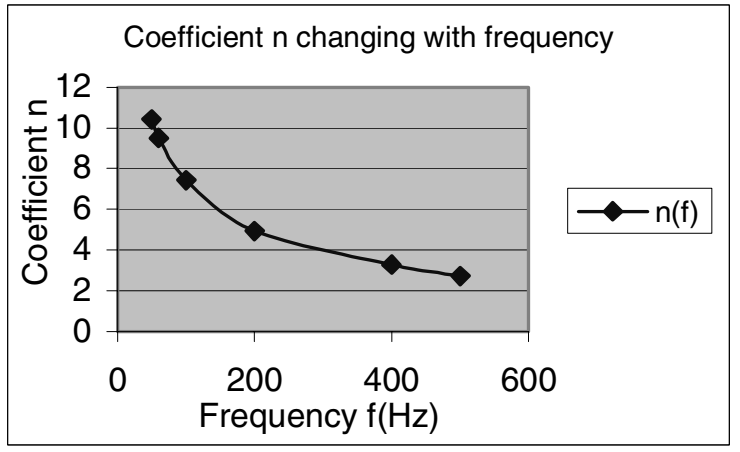

Fig. 12 Coefficient $k_{e}$ changing with frequencies

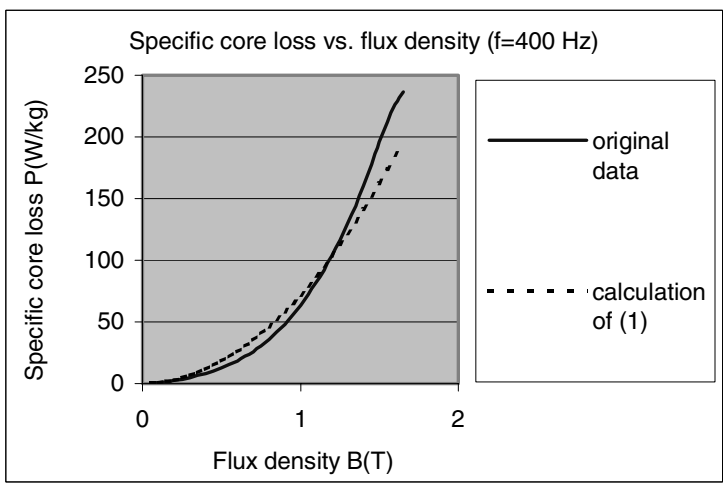

Fig.13 Comparison between the original loss data and the calculation of formula (1) for Mill A annealed material

with frequency. Fig. 10 through to Fig. 12 show these results.

Though these coefficients are mathematically obtained by the minimum technique, they can be explained physically. The eddy current coefficient $k_{e}$ decreases with frequency because of the skin effect, which decreases the conducting area and in turn decreases the eddy current loss. The changes of the hysteresis loss coefficients $k_{h}$ and $n$ with frequency indicate the hysteresis loop area change, which reveals the change of the material domain wall motion.

The above results are applicable to all the investigated electrical steels, including cold rolled motor lamination steels, non-oriented silicon steels, thin-gauge silicon steels, powdered metal alloys and both annealed and un-annealed materials, although the errors between the calculations and the original data are different for different materials. It should be mentioned that the calculation values at 400 Hertz and saturation flux densities are more than the original data for the un-annealed materials, but less for the annealed ones. Fig.13 through to Fig. 15 show the comparison results at 400 Hertz for Mill A annealed material, and Fig.16 through to Fig.18 show the comparison results at 400 Hertz for Mill A unannealed material.

To consider the loss calculation in electrical machines, a 


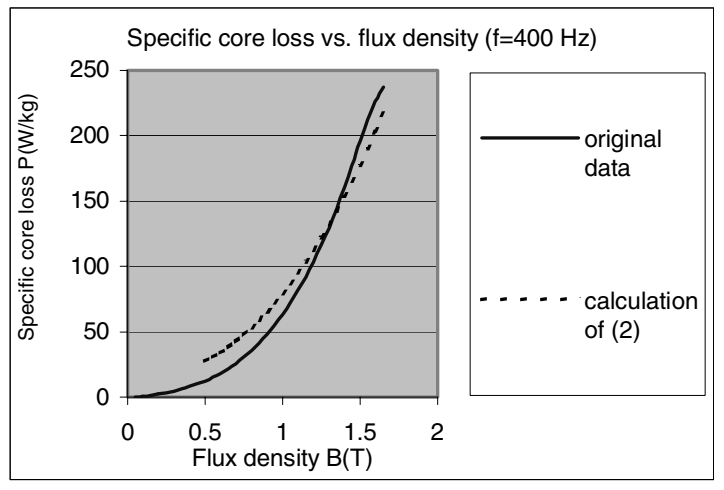

Fig.14 Comparison between the original loss data and the calculation of formula (2) for Mill A annealed material

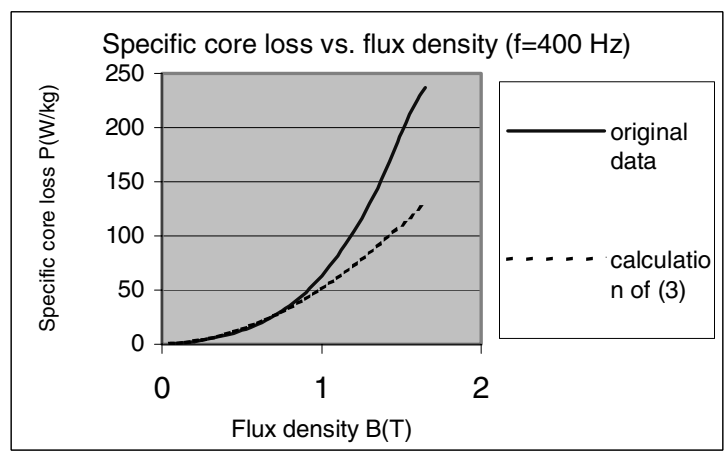

Fig.15 Comparison between the original loss data and the calculation of formula (3) for Mill A annealed material

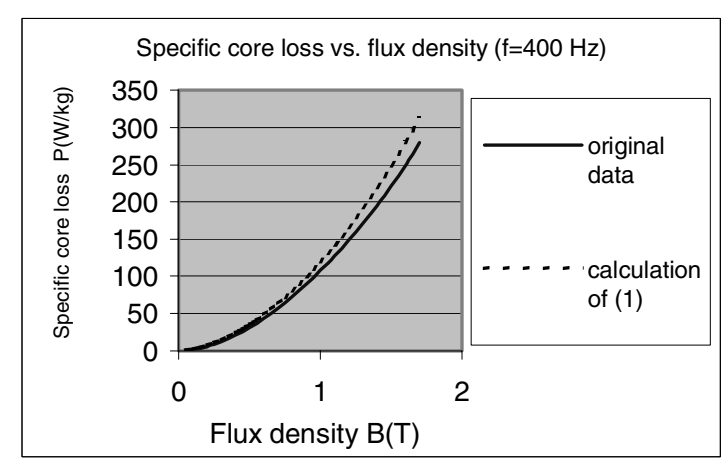

Fig.16 Comparison between the original loss data and the calculation of formula (1) for Mill A un-annealed material

modified three-term formula is proposed as

$$
\begin{aligned}
P_{c} & =P_{h}+P_{e}+P_{a} \\
& =k_{h} f B^{\left(a+b B+c B^{2}\right)}+k_{e} f^{2} B^{2}+k_{a} f^{1.5} B^{1.5},
\end{aligned}
$$

where the constant $n$ in (3) will be replaced by the flux density dependent term $\left(a+b B+c B^{2}\right)$, which represents the difference between the static hysteresis loop and the dynamic hysteresis loop. Here the constant coefficients $a, b$ and $c$ will change with frequency.

To separate the loss in (11), it is divided by frequency to

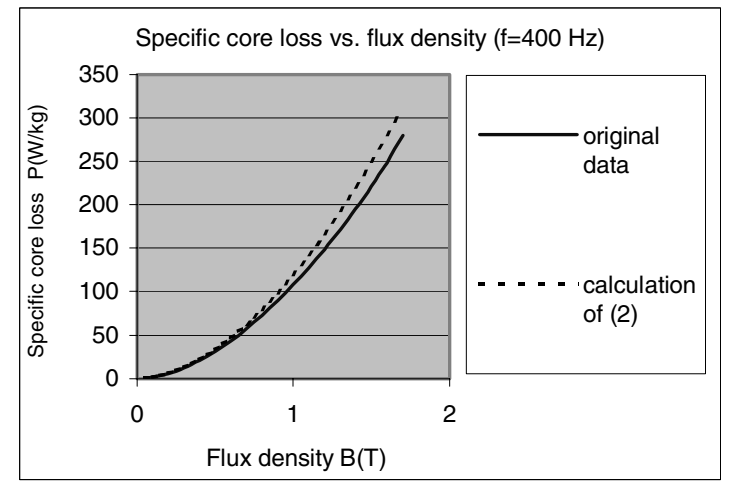

Fig.17 Comparison between the original loss data and the calculation of formula (2) for Mill A un-annealed material

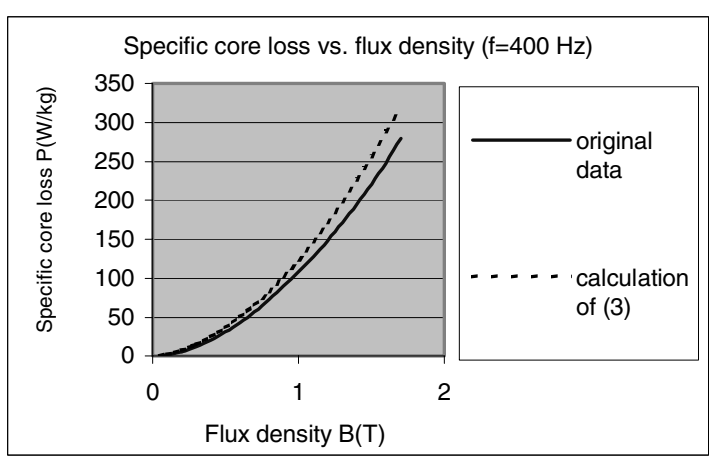

Fig.18 Comparison between the original loss data and the calculation of formula (3) for Mill A un-annealed material

give

$$
\begin{aligned}
P_{c} / f & =P_{h} / f+P_{e} / f+P_{a} / f \\
& =k_{h} B^{\left(a+b B+c B^{2}\right)}+k_{a} \sqrt{f} B^{1.5}+k_{e} f B^{2} .
\end{aligned}
$$

The original core loss data is again used to plot curves of $P_{c} / f$ vs. $\sqrt{f}$ for different values of flux density $B$ from the lowest frequency to the highest frequency. Formula (12) is a parabola in $\sqrt{f}$ and will have the same form as (10), where the coefficients $D, E$ and $G$ can be obtained by way of polynomial curve fitting. The eddy current loss coefficient $k_{e}$ and the excess loss coefficient $k_{a}$ are obtained from $E=k_{e} B^{2}, G=k_{a} B^{1.5}$. These two coefficients are used to separate the hysteresis loss from the total loss as

$$
P_{h}=P_{c}-P_{e}-P_{a},
$$

for different frequencies, which results in the relationship of hysteresis loss versus flux density for each definite frequency, $P_{h}=P_{h}(B)$. Finally, four different hysteresis loss values are chosen to be substituted into the logarithm of $P_{h}=k_{h} f B^{\left(a+b B+c B^{2}\right)}$, giving four simultaneous linear 


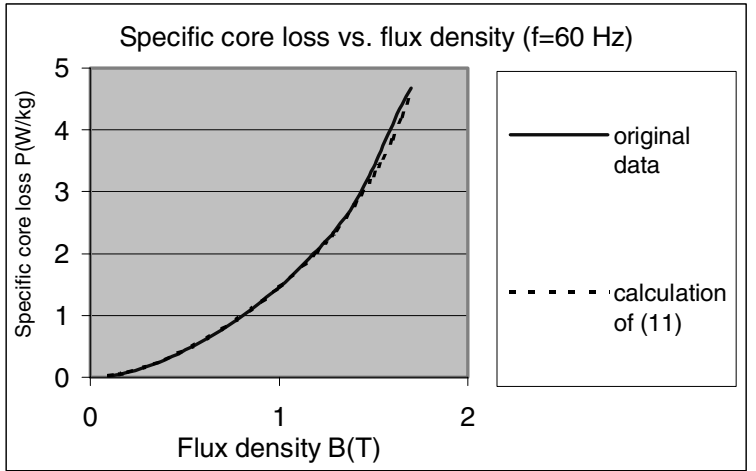

Fig.19 Comparison between the original loss data and the calculation of formula (11) for Ak 26-M47 (60 Hz)

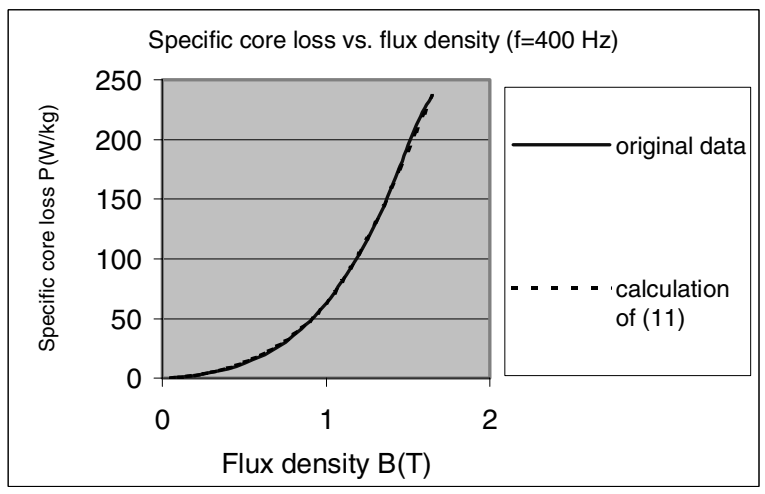

Fig.20 Comparison between the original loss data and the calculation of formula (11) for Mill A annealed material

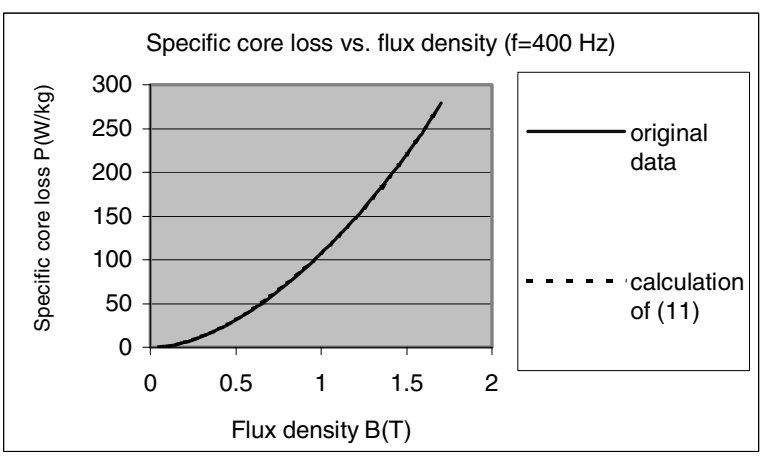

Fig.21 Comparison between the original loss data and the calculation of formula (11) for Mill A un-annealed material

algebraic equations for $k_{h}, a, b$ and $c$ of the form

$$
\log P_{h}=\log k_{h}+\log f+\left(a+b B+c B^{2}\right) \log B .
$$

These are solved for $\log k_{h}, a, b$ and $c$, then $k_{h}$ are obtained from $\log k_{h}$. This process allows for the hysteresis loss coefficients $k_{h}, a, b$ and $c$ to change with frequency, and in turn reveal the hysteresis loop changes. Mathematically, as more data is used to extract the coefficients, the curve fitting between the calculation result of

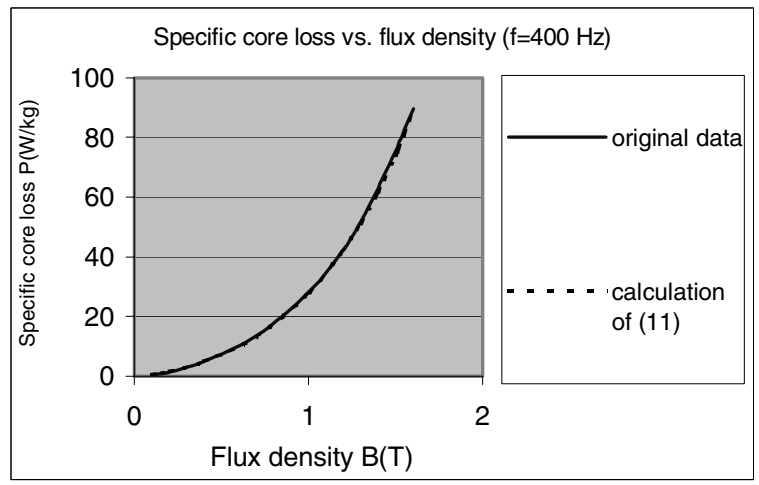

Fig.22 Comparison between the original loss data and the calculation of formula (11) for Ak 26-M47 (400 Hz)

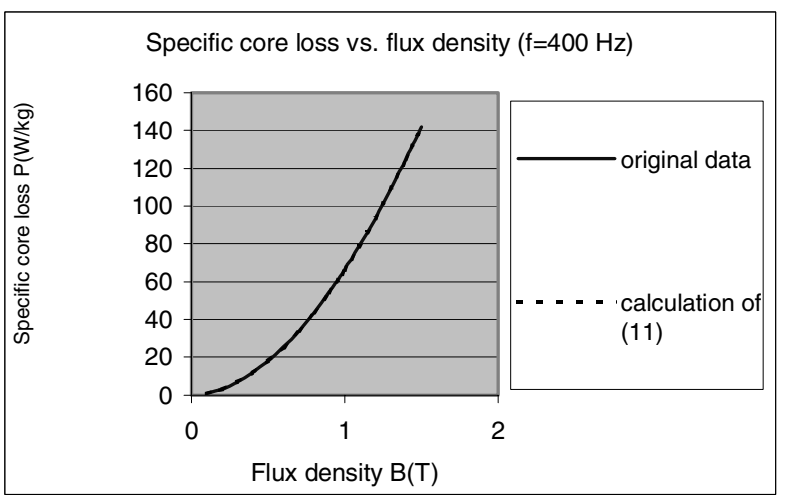

Fig.23 Comparison between the original loss data and the calculation of formula (11) for powdered metal alloy

(11) and the original experimental data will be closer. Fig. 19 and Fig. 22 show the comparison results at both 60 Hertz and 400 Hertz for AK 26-M47. Fig. 20 shows the comparison results at 400 Hertz for Mill A annealed material and Fig.21 shows the comparison results at 400 Hertz for Mill A unannealed material. To extend the application of (11), Fig.23 shows the comparison results at $400 \mathrm{Hertz}$ for the powdered metal alloy.

It can be seen that the match between the calculation of (11) and the original loss data is quite close, which is applicable to both $60 \mathrm{Hertz}$ and $400 \mathrm{Hertz}$, and to all our investigated electrical steels, including cold rolled motor lamination steels, non-oriented silicon steels, thin-gauge silicon steels, powdered metal alloys and both annealed and un-annealed materials. For other frequencies within the range of the original loss data, this formula is also available. It should be mentioned that the eddy current loss coefficient $k_{e}$ and the excess loss coefficient $k_{a}$ are constant in this formula, but the hysteresis loss coefficient $k_{h}, a, b$ and $c$ will change with frequency. 


\section{CONCLUSION}

For electrical machine designers, the core loss data are usually provided in the form of tables or curves of total loss versus flux density or frequency. The currently available loss formulas are limited and not applicable at high flux densities and high frequencies.

The discussion of the loss separation methods and the application of the three loss formulas show that the consideration of dynamic hysteresis loop is very important in core loss calculation. The dependences of these loss coefficients on frequency are presented.

On the basis of the comparison and discussion of the three known formulas, a new modified loss formula for the application in electrical machines is presented. This formula not only represents the three loss terms, hysteresis loss, eddy current loss and excess loss, but also reveals the changes of dynamic hysteresis loop. The extracting method of the loss coefficients and curve fitting results has shown that this formula is both accurate and practical.

\section{ACKNOWLEDGMENT}

The authors acknowledge Black \& Decker, Eaton Corporation, Globe Motors, Ispat Inland, Lamination Specialty Corporation and Small Motor \& Motion Association for assistance with this project.

\section{REFERENCES}

[1] Jianguo Zhu and V.S.Ramsden, "Improved formulations for rotational core losses in rotating electrical machines," IEEE Trans. Magn. vol.. 34, pp. 2234-2242, July 1998.

[2] V.M.Machado and A.L.Ribeiro, "Eddy current hysteresis losses in ferromagnetic media,"“ IEEE Trans. Magn. Vol. .34, pp. 1267-1269, July 1998.

[3] L.R.Dupre, G.Bertotti and J.A.A.Melkebeek, "Dynamic Preisach model and energy dissipation in soft magnetic materials," IEEE Trans. Magn. Vol. .34, pp. 1168-1170, July 1998.

[4] M.Amar and R.Kaczmarek, "A general formula for prediction of iron losses under nonsinusoidal voltage waveform," IEEE Trans. Magn. Vol. .31, pp. 2504-2508, Sept. 1995.

[5] M.amar and F.Protat, "A simple method for the estimation of power losses in silicon iron sheets under alternating pulse voltage excitation," IEEE Trans. Magn. vol.. 30, pp. 842-944, March 1994.
[6] G.Bertotti, F.Fiorillo and M.Pasquale, "Measurement and prediction of dynamic loop shapes and power losses in soft magnetic materials," IEEE Trans. Magn. Vol. .29, pp. .3496-3498, Nov. 1993.

[7] D.C.Jiles, "Frequency dependence of hysteresis curves in "nonconducting' magnetic materials," IEEE Trans. Magn. Vol. 29, pp. .3490-3492, Nov. 1993.

[8] G.Bertotti and M.Pasquale, "Physical interpretation of induction and frequency dependence of power losses in soft magnetic materials," IEEE Trans. Magn. Vol. .28, pp. 2787-2789, Sept. 1992.

[9] G.Bertotti, "Dynamic generation of the scalar Preisach model of hyteresis,” IEEE Trans. Magn. vol.. 28, pp. .2599-2601, July 1992.

[10] H.Pfutzner, P.schonhuber, B.Erbil, G.Harasko and T.Klinger, "Problems of loss separation for crystalline and consolidated amorphous soft magnetic materials," IEEE Trans. Magn. vol. 27, pp. 3426-3431, July 1991.

[11] G.Ban and G.Bertotti, "Frequency and peak induction dependence of eddy current losses in AlSiFe magnetic laminations," IEEE Trans. Magn. Vol. .25, pp..3967-3969, Sept. 1989.

[12] G.Bertotti, "General properties of power losses in soft ferromagnetic materials," IEEE Trans. Magn. Vol. 24, pp. 621-630, January 1988.

[13] G.Bertotti, F.Fiorllo and G.P.Soardo, "Dependence of power losses on peak magnetization and magnetization frequency in Grain-oriented and non-oriented 3\% SiFe," IEEE Trans. Magn. vol. 23, pp. .3520-3522, Sept. 1987.

[14] K.Ohmori and K.I.Arai, "High frequency iron loss of sendust thin ribbons made by a rapid quenching method," IEEE Trans. Magn. vol. 23, pp. 3230-3232, Sept. 1987.

[15] R.H.Gaylord and C.D.Graham, "The frequency of the loss per cycle in ferromagnetic sheet material," IEEE Trans. Magn. vol. 19, pp..35203522, Sept. 1983

[16] Yo Sakaki and Shin-ichi Imagi, "Relationship among eddy current loss, frequency, maximum flux density and a new parameter concerning the number of domain walls in polycrystalline and amorphous soft magnetic materials," IEEE Trans. Magn. vol. 17, pp.1478-1480, July 1981.

[17] S.D.Washko and J.W.Shilling, "Dependence of losses on flux density in grain oriented 3\% silicon steel," IEEE Trans. Magn. vol. 16, pp.737739, Sept. 1980.

[18] K.Narita and M.Imamura, "Frequency dependence of iron losses in 4percent Si-Fe single crystal with (100)[001] orientation," IEEE Trans. Magn. vol. 15, pp.981-988, March 1987.

[19] J.D.Lavers and P.P.Biringeer, "Pridiction od core losses for high flux densities and distorted flux waveforms," IEEE Trans. Magn. vol. 12, pp.1053-1055, Nov. 1976.

[20] A.Ferro, G.Montalenti and G.P.Soardo, "Non linearty anomaly of power losses vs. frequency in various soft magnetic materials," IEEE Trans. Magn. vol. 11, pp.1341-1343, Sept. 1975.

[21] "Switched reluctance motor simulation software," User Manual for PC-SRD 4.7, Motorsoft, INC. 